\title{
A location-based approach to the classification of mesoscale oceanic structures in SeaWiFS and Aqua-MODIS images of Northwest Africa
}

\author{
E. Vidal-Fernández, J. A. Piedra-Fernández, J. Almendros-Jiménez \& M. \\ Cantón-Garbín
}

To cite this article: E. Vidal-Fernández, J. A. Piedra-Fernández, J. Almendros-Jiménez \& M. Cantón-Garbín (2015) A location-based approach to the classification of mesoscale oceanic structures in SeaWiFS and Aqua-MODIS images of Northwest Africa, International Journal of Remote Sensing, 36:24, 6135-6159, DOI: 10.1080/01431161.2015.1111540

To link to this article: http://dx.doi.org/10.1080/01431161.2015.1111540

Published online: 24 Nov 2015.

Submit your article to this journal $\sqsubset$

щ Article views: 88

Q View related articles $\sqsubset$

View Crossmark data ¿

Citing articles: 2 View citing articles $\square$ 


\title{
A location-based approach to the classification of mesoscale oceanic structures in SeaWiFS and Aqua-MODIS images of Northwest Africa
}

\author{
E. Vidal-Fernández, J. A. Piedra-Fernández, J. Almendros-Jiménez*, and \\ M. Cantón-Garbín
}

Department of Informatics, University of Almeria, 04120-Almeria, Spain

(Received 17 April 2015; accepted 11 October 2015)

\begin{abstract}
This study presents a different approach to the classification of Mesoscale Oceanic Structures (MOS) present in the Northwest African area, based on their location. The main improvement stems from the partition of this area in four large zones perfectly differentiated by their morphological characteristics, with attention to seafloor topography and coastal relief. This decomposition makes it easier to recognize structures under adverse conditions, basically the presence of clouds partly hiding them. This is observed particularly well in upwellings, which are usually very large structures with a different morphology and genesis in each zone. This approach not only improves the classification of the upwellings, but also makes it possible to analyse changes in the MOS over time, thereby improving the prediction of its morphological evolution. To identify and label the MOS classified in the Sea-viewing Wide Field-of-view Sensor (SeaWiFS) and Aqua-MODIS (Moderate Resolution Imaging Spectroradiometer) chlorophyll- $a$ and temperature images, we used a tool specifically designed by our group for this purpose and which has again shown its validity in this new proposal.
\end{abstract}

\section{Introduction}

Since the mid-seventies, satellite ocean observation has provided a huge amount of valuable data on physical and biological oceanography. This information, stored in the enormous databases of space and environmental agencies, is indispensable for analysing the growing number of environmental problems (Climate Change (2013); Gregg et al. (2003)). Global ocean circulation, most of which is mesoscale (spatial scale of $50-500 \mathrm{~km}$, time scale of 10-100 days), plays an essential role in global climate. In this sense, the energy of Mesoscale Oceanic Structures (MOS), which is at least one order of magnitude higher than general circulation, makes their study determinant in understanding ocean dynamics and analysing the alarming global change (Rubino (2005); Angel and Fasham (1983)).

The zone selected for our study, the Northwest African area, presents conditions appropriate for the formation of MOS, such as upwellings, filaments, cold and warm eddies, and wakes, throughout the year (García-Weil et al. (2000); Barton et al. (1998); Arístegui et al. (1994); Sangra et al. (2005); Tejera et al. (2002); Meunier et al. (2012)) (see Figure 1).

Of all of the MOS present in the zone of interest, the coastal upwelling is undoubtedly worthy of special attention, since studies (Brink (1983); Van-Camp et al. (1991); Nykjaer and Van-Camp (1994); Hernández-Guerra, and Nykjaer (1997)) have shown the existence

*Corresponding author. Email: jalmen@ual.es 


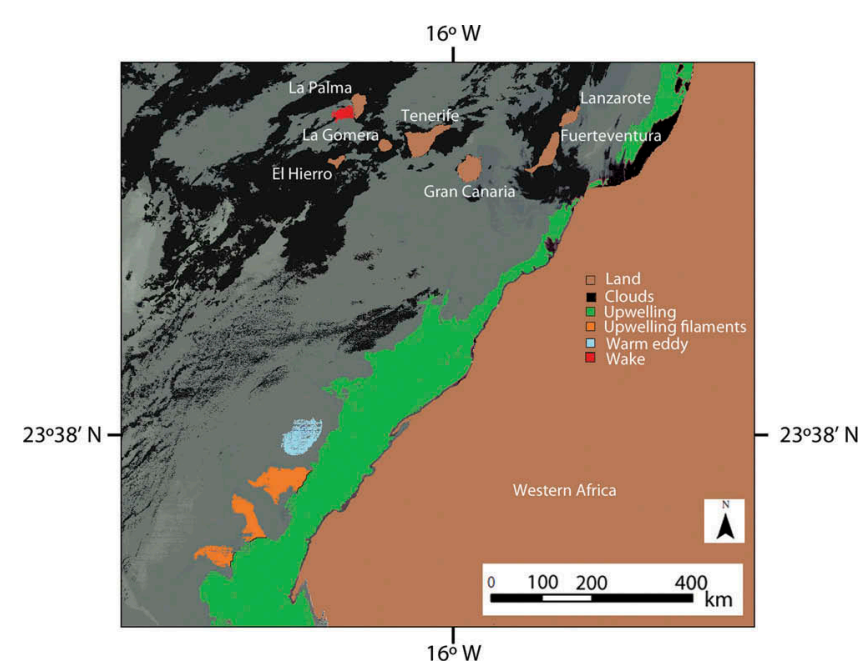

Figure 1. Aqua-MODIS SST scene (24 March 2003). Ocean structure map where upwellings have been coloured in green for visualization, upwelling filaments (Cap Blanc) in orange, warm eddies in light blue, and wakes (west of La Palma island) in red.

of upwelling centres located along the Northwest African coast, specifically off Cape Ghir, Cape Bojador, and Cap Blanc (Ras Nouadhibou). These centres are due to the different wind conditions, variability in coastal topography, and the continental platform, giving rise to four well-differentiated zones along it delimited by the coasts' largest capes (see Figure 2), as proposed in García-Weil (1998); Garcia-Weil et al. (2000); Barton et al. (1998); Nieto, Demarcq, and McClatchie (2012), although with different limits. It so happens that there is frequent cloud cover in that zone, which causes the upwelling to be partially covered by clouds in most of the satellite images processed, leaving only pieces of it in view. At other times, upwelling genesis, more or less active depending on the season, causes it to be present in only one zone. A system that not only segments and identifies the MOS present in the zone, but could also classify those pieces of upwelling according to their location in one of those zones would make recognition, study, and prediction of the evolution of their morphology easier for experts. We should mention that, for instance, filamentous structures detached from the upwellings under certain conditions can be identified by their morphology and other external factors. This article presents an improvement over our previous work (Vidal-Fernández et al. (2015)), creating a system able to segment and identify the MOS in the four new areas of the upwelling, achieving high accuracy in recognition of the new regions identified, and allowing a more precise study of their evolution by queries on the area of interest.

A coastal upwelling (see Figure 1) occurs when dense cold water at the ocean bottom rises towards the coast, transporting nutrients that allow phytoplanktons to develop and proliferate. In our study area, they originate from the action of trade winds from the NE on the sea's surface and the Coriolis Effect with a usually abrupt transition between upwelled waters and the rest of the surface waters in the form of fronts (Barton et al. (1998)). Their analysis and prediction are very important for commercial fisheries, and satellite images provide a powerful tool for their study. Therefore, the improved analysis and recognition proposed in this article are significant improvements in their location and monitoring. 


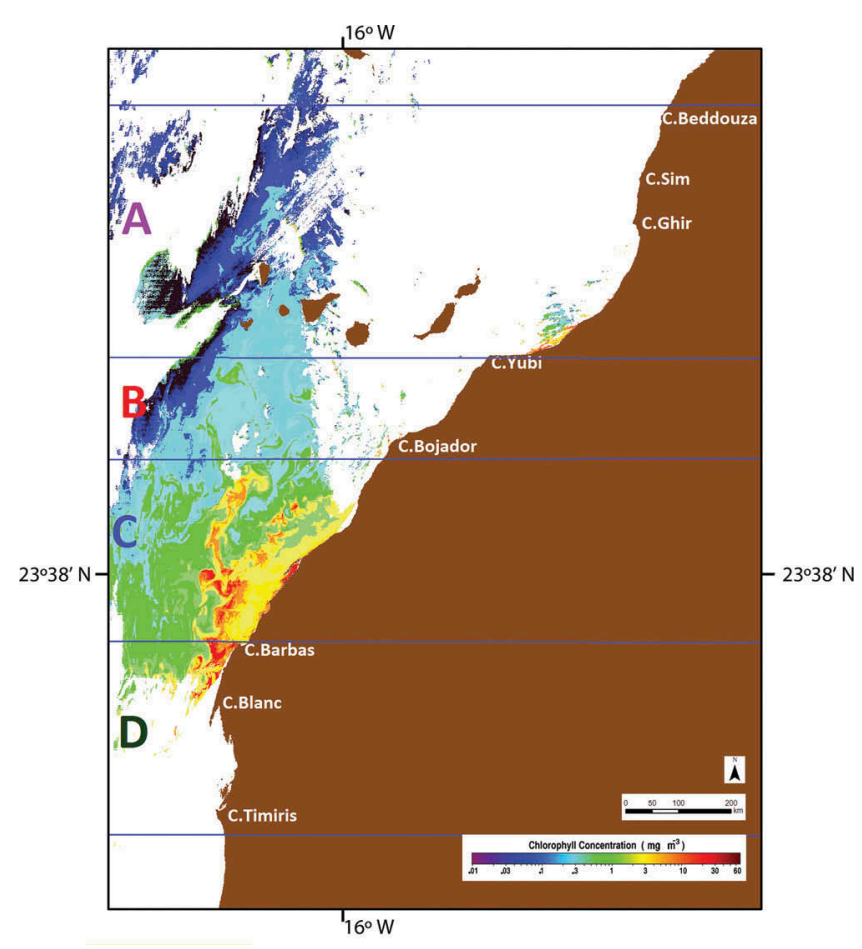

Figure 2. Aqua-MODIS OC scene (9 February 2004). Proposed partition of Northwest African area in four zones (A, B, C, and D). The limits are defined by Cape Beddouza $\left(32^{\circ} 30^{\prime} \mathrm{N}\right)$, Cape Jubi $\left(28^{\circ} \mathrm{N}\right)$, Cape Bojador $\left(25^{\circ} 30^{\prime} \mathrm{N}\right)$, Cape Barbas $\left(22^{\circ} \mathrm{N}\right)$, and Cape Timiris $\left(19^{\circ} \mathrm{N}\right)$.

Upwelling filaments (see Figure 1) emerge from coastal upwellings in the form of tongue-shaped cold water, which extend hundreds of kilometres out to the sea. They are important coast-to-open sea nutrient carriers often occurring near large capes (Meunier et al. (2012)). Being able to identify and locate the specific zone they occur is another of the goals that can improve the classification proposed.

Eddies are more or less round structures that appear near islands or in the open ocean. In cool eddies, cold nutrient-rich water rises to the surface in counterclockwise swirls (Tejera et al. (2002)). The opposite, warm eddies (see Figure 1), drag water with organic matter down to the ocean floor, leaving warm water on the surface, and are formed under stronger wind conditions. The water in the eddies differs from the surrounding water in salinity and temperature, and can travel long distances for long periods of time without mixing with the water that surrounds it (Arístegui et al. (1994); Sangra et al. (2005)).

Wakes (see Figure 1) are warm oceanic structures associated with islands (Barton et al. (1998); Arístegui et al. (1994)). In our study zone, these structures are generated by the impact of predominant winds from the NE on the highest volcanic islands in the archipelago, mainly the western ones. This interaction forms thin tongues of warm water poor in nutrients compared with the water surrounding it and leeward of the islands.

This article presents a location-based approach to the classification of MOS in Seaviewing Wide Field-of-view Sensor (SeaWiFS) and Aqua-MODIS (Moderate Resolution Imaging Spectroradiometer) sensor satellite images of the Northwest 
African area, segmented and identified by our system (Vidal-Fernández et al. (2015)), an object-based image analysis (OBIA)-based method that takes hierarchical data format (HDF) files as input, processes the image, and segments them into regions, calculating a series of descriptors for each for their later classification in the categories proposed. In this article, we intended to go one step further, improving the classification presented in Vidal-Fernández et al. (2015). To carry this out, the morphological diversity of the MOS classified in the study area was analysed. Since there are four zones of interest defined by the seafloor topography and coastal relief, it is possible to visualize well-differentiated characteristic morphological patterns for each of the MOS, especially upwellings, because of the large areas they occupy in the ocean along the coast. Furthermore, another factor to consider for classification is the adverse weather conditions, basically due to clouds that partly hide the regions or result in noise, which impedes recognition. It was therefore decided to make an MOS subclassification within these four zones of interest to improve the accuracy rate of the classifiers used. The goal is not only to improve the accuracy rate, but also to provide a valuable tool for experts in the subject for analysing MOS, and specifically, the upwelling along the Northwest African coast, both overall and by area. This enables, among other things, an observation of their evolution over time and finding out their persistence and morphological variations arising over time. To carry out this work and determine conclusive results, a significant sample of images containing MOS must be available, so our system is able to process thousands of images automatically, identifying the MOS present in them.

\section{Material and methods}

\subsection{Satellite data and area of interest}

The oceanic satellite images of chlorophyll- $a$ (referred to as 'OC' - i.e. the quantity of chl- $a$ contained in the ocean) from SeaWiFS (on board the Orbview-2 satellite. $1.1 \mathrm{~km}$ resolution) and Aqua-MODIS (on board the Aqua satellite. $1.1 \mathrm{~km}$ resolution), and temperature (sea surface temperature, SST), also from the Aqua-MODIS sensor, are a stupendous choice for the study of MOS (Liu and Hou (2012); Patel, Balasubramanian, and Gangopadhyay (2008); Mityagina and Lavrova (2008); Marcello, Marques, and Eugenio (2005)) for primary production (Xiao, Hu, and Qiang (2010)) and global change (Sathyendranath et al. (2012)) in any part of the world. In situ measurements in the study area were compared (Saulquin, Gohin, and Garrello (2011)) with chl- $a$ image data from these sensors over a 10 year period, and correlation coefficients were very high, as they were for comparison of the different sensors with each other.

In view of the above, we used OC and SST images from these two sensors (SeaWiFS and Aqua-MODIS), acquired free of charge from the NASA Web Ocean Color http://oceancolor.gsfc.nasa.gov in 2013, for our classification to improve analysis. A total of 212 satellite images from the Canary Island Archipelago and Northwest African area (the geographic centre of the Islands is at coordinates latitude $28^{\circ} 11^{\prime} 60^{\prime \prime} \mathrm{N}$ and longitude $16^{\circ} 23^{\prime} 60^{\prime \prime} \mathrm{W}$ ) were processed. Of them, 92 were chl- $a$ images from the SeaWiFS sensor taken when it was active in this zone (1997-2004). The images are derived from Level L2 Merged Local Area Coverage (MLAC) products (with radiometric calibration and geometric correction). The other 120 are Aqua-MODIS sensor images from 2003 to 2013, comprising of $61 \mathrm{chl}-a$ images and 59 temperature images. These images were derived from Level L2 Local Area Coverage (LAC) products. 
A total of 365 MOS were labelled, of which 284 were upwellings or pieces of upwellings (because of cloud cover, it is impossible to completely segment the upwelling in a single region, and so it is divided into two or more pieces), 44 upwelling filaments, 10 cool eddies, four warm eddies, and 23 wakes.

Figures 4 and 5 show examples of segmented structures identified from SeaWiFS, Aqua-MODIS OC, and Aqua-MODIS SST sensors.

\subsection{OBIA MOS identification system}

To carry out an in-depth study of ocean dynamics, there must be automatic MOS regional detection and identification tools available to process oceanic satellite images and enable their later classification by criteria that facilitate the work of oceanographers, e.g. to locate the MOS, especially upwellings and upwelling filaments, and to study their evolution patterns. It is extremely useful to work on a regional level and not an image pixel level for this, and hence we based our tool design on OBIA (Blaschke (2010)). The final purpose of an OBIA system is to clearly identify the objects of interest (MOS, in our case) based on some primitive objects (initial regions resulting from segmentation), which evolve into objects of interest (final regions) by incorporating expert knowledge, as explained in Baatz, Hoffmann, and Willhauck (2008), or by using specific segmentation techniques for each object class, as in Musci, Feitosa, and Costa (2013). Some examples of image recall techniques and tools based on the use of regions are discussed in Shrivastava and Tyagi (2014) and Ko, Yonsei, and Byun (2005).

Apart from this, OBIA techniques are appropriate, because MOS are characterized by their wide morphological and contextual variability, as highlighted by authors who have applied this technique for analysing scenarios highly variable in space and time (Rastner et al. (2014); Dragut, and Blaschke (2006); Jovanovic et al. (2010)). In fact, the images used to evaluate our tool span a wide time range (1997-2013), with clouds partially covering the structures and making the MOS completely different in each one. Based on this, our tool was designed to be able to detect and combine regions by individual MOS characteristics and experts' recognition rules.

We used the tool designed by our research group (Vidal-Fernández et al. (2015)), which takes HDF format files as input, automatically processes the images, and segments the regions. Only those images that meet the usability criteria go on to the next stage: data must correspond to the geographic zone of study (Northwest African area in this case) and over $20 \%$ of the pixels in the whole image (those that are not clouds or errors) must be useful. It was implemented because the system enables hundreds of images to be loaded at a time without any need for previewing them. After a global automatic preprocessing stage (using a smoothing filter-double median filter applied to the greyscale image to eliminate noise and loose pixels), the system segments the images into primary regions, using thresholding and edge detection. The image is thresholded in a range of grey values defined to configure a greyscale representing homogenous information. For us, each one of these ranges of grey values represents exactly $1^{\circ} \mathrm{C}$ in the SST or a certain quantity of chl- $a\left(\mathrm{mg} \mathrm{m}^{-3}\right)$, depending on whether it is a temperature or chl- $a$ image. Thresholding returns a binary image of the region to be segmented. A morphological opening operation is applied to it (diffusing edges, eliminating bumps, and separating regions joined by narrow points) and an edge-detection technique (polygonal approximation algorithm) is applied to determine the region contour. After this stage the system extracts the 

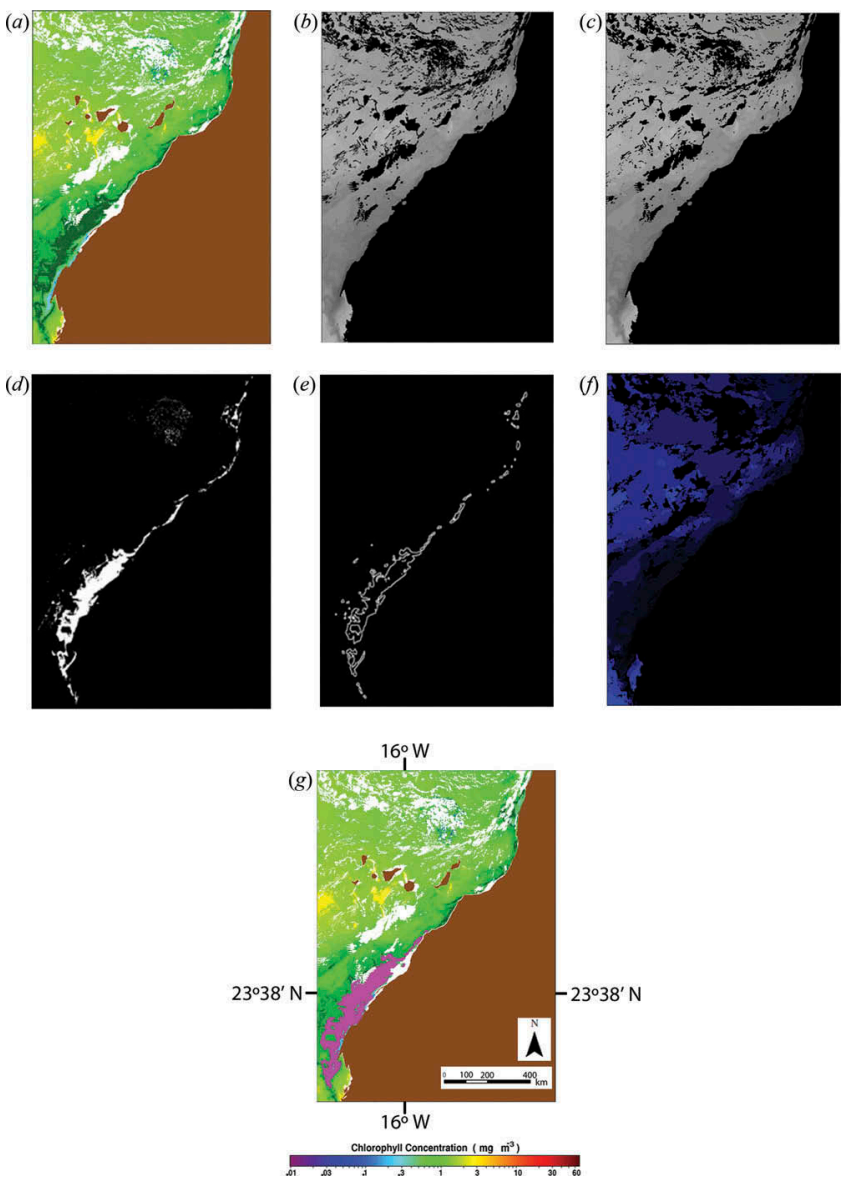

Figure 3. Aqua-MODIS SST scene (22 September 2008). (a) Original SST image in colour. (b) Original SST image in greyscale. (c) Image after preproccesing stage. (d) Example of the results of thresholding. (e) Example of the results after detecting the edges of that threshold. $(f)$ Final mask image after segmentation. $(g)$ Image resulting from labelling an upwelling.

region descriptors automatically. Figure 3 shows the images resulting from each processing stage.

To solve the problem of cloud cover or noise that could make a region corresponding to an MOS appear broken up in the image, the tool improves region detection by combining with others nearby (the system allows a 0 - to 20 -pixel proximity tolerance to be selected) with similar chl- $a$ or SST characteristics. The information on the regions is automatically stored in a relational $\mathrm{x}$ designed to store not only regional data, but also the image it comes from, the sensor, and the satellite. Queries to the region database are made for a set of diffuse descriptors (spectral, morphological, and contextual) calculated during processing.

Sixty-four descriptors were calculated and stored for each of the single, or combined, regions: Hu moments: Hu1,..,Hu7 (Hu (1962)); Maitra moments: MM1,..,MM6 (Maitra (1979)); Tensorial moments: M00, M01, M02, M03, M10, M11, M12, M20, M21, and M30 (Galvez, and Cantón (1993)); and Zernike moments: MZ1,...,MZ6, MP1, and MP2 (Teague 
(1980)); Perimeter and Area; descriptors related to BoundingEllipse (i.e. the ellipse that contains the region): Circularity, Eccentricity, MajorAxis, MinorAxis (Zunic, and Slado (2000)), Centroid $x$, Centroid_y, Cirscumpcription (value of zero means ellipse is out and one means ellipse is completely on the region), and Orientation; descriptors related to BoundingBox (i.e. the rectangle which contains the region): BBox_height, BBox_width, BBox_coordinate_ $x$, and BBox_coordinate_y; and diffuse descriptors related to chl- $a$ concentration (chl- $a$ _very_low, chl- $a$ _low, chl- $a$ _medium, chl $a$ _high, and chl- $a$ _very_high), temperature (SST_very_cold, SST_cold, SST_hot, SST_warm, and SST_very_hot), distance from continent (Cont_dist_near, Cont_dist_medium, and Cont_dist_far), distance from island (Island_dist_near, Island_dist_medium, and Island_dist_far), and size (Size_small, Size_medium, and Size_large). All of these values are used during classification.

\subsection{MOS classification proposals. Relationship with four zones in the Northwest African area}

The last stage of our OBIA system (Vidal-Fernández et al. (2015)) consists of automatically classifying the final regions of interest found as one of the MOS we are interested in using the descriptors above: Upwelling (U), Upwelling Filament (UF), Cool Eddy (CE), Warm Eddy (WE), or Wake (W). Therefore, a first classification proposal would be to define five MOS categories, one for each type: U, UF, CE, WE, and W, results of which were analysed in Vidal-Fernández et al. (2015).

It should be mentioned that because of the morphological variability in the upwelling due to marine topography, the coastal relief, and other factors, such as wind and ocean currents, it is especially difficult to recognize these structures, and furthermore, the presence of clouds impedes classification even more. The Northwest African coast has special characteristics that makes it possible to divide it into four zones (García-Weil (1998)) (see Figure 2).

- Zone A: Region between Cape Beddouza $\left(32^{\circ} 30^{\prime} \mathrm{N}\right)$ and Cape Juby $\left(28^{\circ} \mathrm{N}\right)$, including Cape Ghir $\left(30^{\circ} 30^{\prime} \mathrm{N}\right)$. It is characterized by irregular undersea topography and the geometry and topography of the coast. The upwelling centre is active all year long, with maximums in summer-autumn, and the Cape Ghir upwelling filament, which can be as much as 20-50 km wide, extends seaward 100-300 km (Nykjaer (1988); Hernandez-Guerra and Nykjaer (1997)).

- Zone B: Region between Cape Juby $\left(28^{\circ} \mathrm{N}\right)$ and Cape Bojador $\left(25^{\circ} 30^{\prime} \mathrm{N}\right)$, characterized by a narrow, abrupt continental platform, with an irregular coastal geometry. Cape Juby and Bojador are outstanding. It includes the Canary Island Archipelago and the formation of wakes can be observed to their southwest. The upwelling is not very long, but upwelling filaments near Cape Bojador (Van-Camp et al. (1991)) and cyclonic and anticyclonic eddies to the south of the Islands (Barton et al. (1998); Arístegui et al. (1994)) stand out.

- Zone C: Region between Cape Bojador $\left(25^{\circ} 30^{\prime} \mathrm{N}\right)$ and Cape Barbas $\left(22^{\circ} \mathrm{N}\right)$, characterized by a wide gently sloping continental platform and coastal geometry without appreciable irregularities. The upwelling is present all year long, with maximums in spring and autumn, and upwelling filaments may extend from 150 to $200 \mathrm{~km}$ out to the sea. There are also anticyclonic eddies of up to $100 \mathrm{~km}$ in diameter, basically towards the beginning and end of the year (García-Weil (1998)).

- Zone D: Region between Cape Barbas $\left(22^{\circ} \mathrm{N}\right)$ and Cape Timiris $\left(19^{\circ} \mathrm{N}\right)$, including Cap Blanc $\left(21^{\circ} \mathrm{N}\right)$. Its characteristics make the conditions north of Cap Blanc 
propitious for the upwelling all year long. To the south there are periods without any upwelling. The Cap Blanc upwelling filament, which may extend up to $400 \mathrm{~km}$ out to the sea, and anticyclonic eddies are outstanding (Van-Camp et al. (1991); Hernandez-Guerra and Nykjaer (1997)).

Since most of the time only pieces of upwellings are found and not the entire upwelling covering the whole study area (normally due to clouds or because it is a period of low activity and it only appears concentrated at the upwelling centres), it seems reasonable to attempt to classify the upwellings based on their location, the zone they span, with attention to the four zones mentioned (see Figure 2). It would also be useful for the expert community to locate the upwelling filaments near Cape Ghir or Cap Blanc. Extraordinarily long filaments (at least $200 \mathrm{~km}$ offshore), which are important vehicles for nutrient and carbon transport in that zone, are recurrent at Cabo Ghir (Troupin et al. (2012)). The filaments and eddies are basically generated by interaction of the shape of the coast and the seafloor bathymetry in their interaction with the wind, and this factor is predominant in their generation. At Cabo Ghir a cyclonic eddy is also generated. Generation of a filament (over $280 \mathrm{~km}$ ) is observed at Cape Blanc, the genesis and surroundings of which may be a little more complex than at Cape Ghir. Some authors (Troupin et al. (2012)) suggest that some filaments, in this case the one at Cape Blanc, may be caused by the interaction between an outside eddy field and upwelling water (Meunier et al. (2012)). Therefore, a new classification proposal would have 15 categories.

- Ten upwelling categories, by the area or areas they span either entirely or partly. It should be noted that after segmentation it is unusual to find the upwelling, or pieces of it (because of being partly covered by clouds), limited to only one of the four zones defined, and what is normally found in practice is upwellings or pieces of upwelling that span two or more, and even four zones:

○ UA - Upwelling spans all or part of Zone A

- UB - Upwelling spans all or part of Zone B

- UC - Upwelling spans all or part of Zone C

○ UD - Upwelling spans all or part of Zone D

○ UAB - Upwelling partly or completely spans Zones A and B

- UBC - Upwelling partly or completely spans Zones B and C

- UCD - Upwelling partly or completely spans Zones C and D

- UABC - Upwelling partly or completely spans Zones A, B, and C

- UBCD - Upwelling partly or completely spans Zones B, C, and D

- UABCD - Upwelling partly or completely spans four Zones, A B, C, and D

- Two Upwelling Filaments categories, depending on whether the location is farther north (Zones A and B) or south (Zones C and D) of the zone of interest:

- UFAB - Upwelling Filament located on the strip made up of Regions A and B

$\circ$ UFCD - Upwelling Filament located on the strip made up of Regions C and D

- Cool Eddy

- Warm Eddy

- Wake

Figure 4 shows examples of upwellings for each of the 10 subcategories defined (labelled regions have been coloured in black for visualization; previously they were coloured in red), and Figure 5 shows examples of the rest of the MOS: two categories of Upwelling 


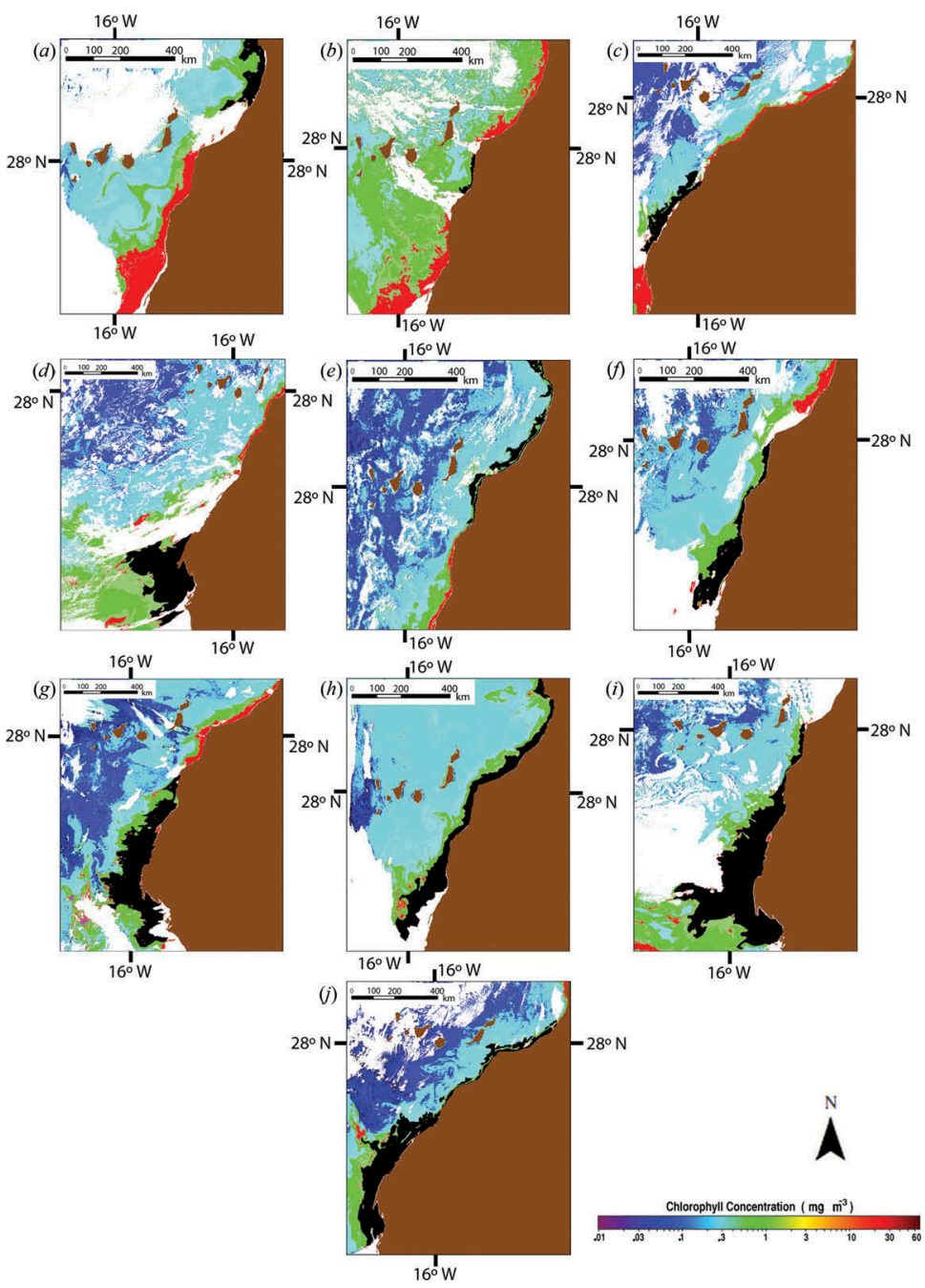

Figure 4. Segmented and labelled upwellings (the regions of interest have been coloured in black for visualization; previously they were coloured in red) from SeaWiFS sensor images (SW) and Aqua-MODIS OC sensor images (MOC). (a) UA. SW (15 July 2001). (b) UB. SW (27 January 1999). (c) UC. MOC (29 October 2009). (d) UD. MOC (20 October 2008). (e) UAB. SW (7 October 1997). (f) UBC. SW (20 September 2003). (g) UCD. SW (9 November 2002). (h) UABC. SW (21 March 2002). (i) UBCD. SW (27 September 2004). (j) UABCD. MOC (4 October 2009).

Filament defined, Cool Eddy, Warm Eddy, and Wake (labelled region contours have been coloured in black for visualization).

\subsection{Classification algorithms}

We should mention that a selection of descriptors was made for the study and different classifiers were applied. As expected, the same set of characteristics does not lead to the same results when working with a whole upwelling as with one of the four upwelling areas described. The training process from regions and selected descriptors uses learning 


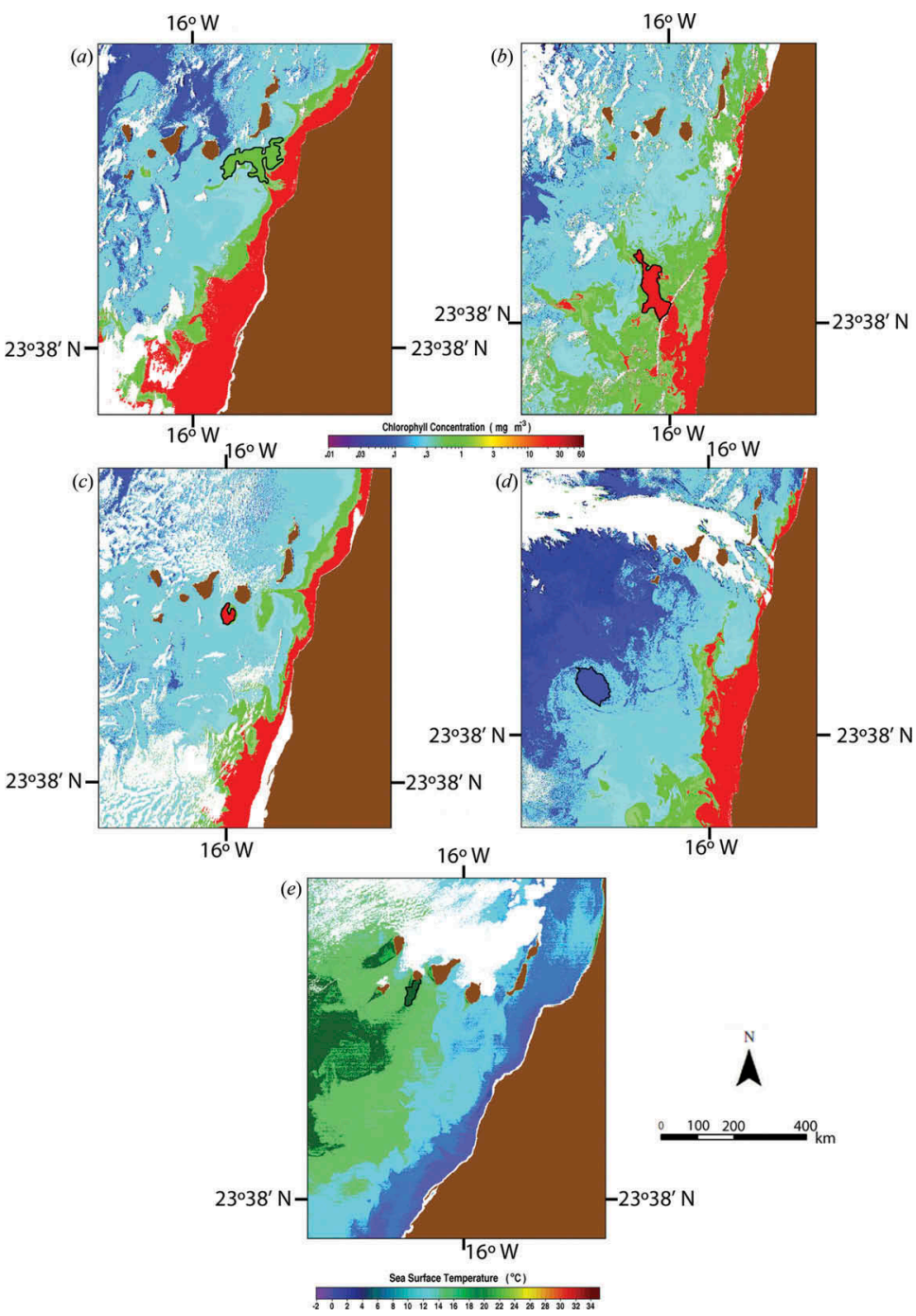

Figure 5. Segmented and labelled regions (the region of interest contours have been coloured in black for visualization). SeaWiFS sensor images: (a) UFAB (26 July 1999), (b) UFCD (29 January 2004), (c) CE (9 July 2004), and (d) WE (22 November 2004). Aqua-MODIS SST sensor image: (e) W-La Gomera island (17 March 2004).

algorithms. We have selected five classifiers based on their good results reported in the literature and low computational cost. The selected classifiers were: C4.5 algorithm (decision tree) (Quinlan (1993)), Naïve Bayes classifier (Bayesian network) (John and Langley (1995)), multilayer perceptron (MLP) (artificial neural network) (Minsky and Papert (1972)), $k$-nearest neighbours classifier ( $k$-NN algorithm) (Aha, and Kibler (1991)), and the improved $k$-NN algorithm based on genetic algorithms, NNge (Brent (1995)). The Cross-validation Folds10-Seed 1 test option was used for all classification algorithms (Kohavi (1995)).

The Correlation-based Feature Selection (CFS) algorithm (Hall (1998)) was used for the selection of classification descriptors. This algorithm shows the degree of 
relevance (near 100\%) or irrelevance (near $0 \%$ ) of the descriptor for the classification. The selection criterion, used in all tests conducted, was to choose all of the descriptors with over $60 \%$ relevance in each case. This gave us high rates of accuracy when there were a small number of descriptors, compared with the total descriptors available (64).

The OBIA system was presented in our previous study (Vidal-Fernández et al. (2015)), specifically designed by our group for the identification of MOS; it explained the different parts from a methodological and functional viewpoint, and demonstrated its validity based on some simple experiments. The results showed that the descriptors, calculated for each of the MOS identified and labelled by the system, enabled the automatic classification of the MOS in the five basic categories (upwelling, upwelling filament, cool eddy, warm eddy, and wake), with a high degree of correspondence between what we labelled manually and what the system classified automatically. To improve the classification, the study area was divided into four regions of interest, and new subclasses were created from the five basic classes. Thus by considering the seafloor topography, coastal relief, and adverse weather conditions, we were able to improve the MOS identification.

\section{Results and discussion}

We started out from a set of regions already segmented with our tool (Vidal-Fernández et al. (2015)) and labelled by hand. This labelling was validated by an oceanographer. A total of 365 MOS were classified into 15 subcategories, of which 284 were upwellings or pieces of upwellings (that could not be combined to form a complete one), divided into a total of 10 subcategories, 44 upwelling filaments, divided into two subcategories, 10 cool eddies, four warm eddies, and 23 wakes. These regions pertained to images from different imageries: SeaWiFS OC, Aqua-MODIS OC, and Aqua-MODIS SST. Table 1 specifies the labelled structures, showing those pertaining to the 15 subcategories by the original sensor image.

Table 1. Number of labelled MOS for each sensor.

\begin{tabular}{lccrr}
\hline MOS & SeaWiFS OC & MODIS OC & MODIS SST & Total \\
\hline UA: Upwelling Region A & 14 & 16 & 17 & 47 \\
UB: Upwelling Region B & 1 & 1 & 1 & 3 \\
UC: Upwelling Region C & 4 & 5 & 6 & 15 \\
UD: Upwelling Region D & 2 & 2 & 2 & 6 \\
UAB: Upwelling Region AB & 17 & 11 & 6 & 34 \\
UBC: Upwelling Region BC & 7 & 2 & 7 & 16 \\
UCD: Upwelling Region CD & 17 & 10 & 11 & 38 \\
UABC: Upwelling Region ABC & 16 & 12 & 6 & 34 \\
UBCD: Upwelling Region BCD & 7 & 9 & 11 & 27 \\
UABCD: Upwelling Region ABCD & 37 & 11 & 16 & 64 \\
UFAB: Upwelling Filament Region AB & 9 & 3 & 1 & 13 \\
UFCD: Upwelling Filament Region CD & 14 & 11 & 6 & 31 \\
CE: Cool Eddy & 8 & 1 & 1 & 10 \\
WE: Warm Eddy & 2 & 1 & 1 & 4 \\
W: Wake & 3 & 1 & 19 & 23 \\
Total (U+UF+CE+WE+W) & 158 & 96 & 111 & 365 \\
\hline
\end{tabular}


The fact that we had such a small sample of cool eddies, warm eddies, and wakes was due basically to the cloud conditions in the area of study and to the small size of these structures, which impedes their clear identification; hence, after validation by experts, many of those that were seriously doubtful were discarded. Logically, in the case of warm wakes, there was an additional problem of their being extremely difficult to find in OC images; however, since they are easier to find in SST images, the sample is larger in that case.

The classification was tested with final regions. The regions were grouped to carry out six tests, for each one of the sensors separately, and for these other three groups: OC sensors (SeaWiFS and MODIS OC), MODIS (OC + SST), and all the final regions labelled and validated irrespective of what the sensor was (SeaWiFS, MODIS OC, and MODIS SST).

To determine the goodness of the division into four zones of the area of interest, we carried out a first test in which only those regions of the upwelling pertaining to categories UA, UB, UC, and UD were selected, i.e. upwelling or pieces of upwelling limited to only one of the four zones defined in Section 2.3. The results could not be better, since the accuracy rate was $100 \%$ or nearly so in each category. The descriptors selected were M11, MM4, BBox_height, BBox_coordinate_y, Orientation, and Size_small. This made us believe that the idea of dividing the area of interest into four zones is good, not only because it corresponds to particular topographic and geographic characteristics that intervene in the genesis of the MOS in those zones, but also because the results in the ideal case of finding only this type of region, i.e. upwellings or pieces of them that only take up a certain zone, support this decision. The problem is that, as we noted in Section 2.3, normally upwellings or the pieces found span more than one zone at a time, and hence the results must be analysed keeping this reality in mind. At the same time, not only are upwellings found in the study area, our system can also segment Upwelling Filaments, Cool Eddies, Warm Eddies, and Wakes. Since we were pursuing automation of the complete process, we had to include these structures in our analysis as well, and check the final classification performance of our system. The analysis of all these structures is fundamental to study ocean dynamics, and thus their identification is quite important.

Table 2 lists the accuracy rates found employing the different classification algorithms for the 15 categories of MOS proposed in Section 2.3 (UA, UB, UC, UD, UAB, UBC, $\mathrm{UCD}, \mathrm{UABC}, \mathrm{UBCD}, \mathrm{UABCD}, \mathrm{UFAB}, \mathrm{UFCD}, \mathrm{CE}, \mathrm{WE}$, and $\mathrm{W}$ ).

The first thing we observed was that the accuracy rates were not near $100 \%$. This was because there was no longer just the ideal upwelling limited to only one of the four zones, but other structures as well within diffuse margins of belonging to some of the categories proposed. Moreover, such a small sample of UB and UD, along with the already reduced sample of cool eddies, warm eddies, and wakes, made the results modest. As we did not

Table 2. Results of MOS classification with the six tests for the 15 categories. All figures shown are percentages.

\begin{tabular}{lcccccc}
\hline Algorithm & SW & M-OC & M-SST & SWOC & MOD & All \\
\hline C 4.5 & 72.78 & 48.96 & $\mathbf{6 3 . 9 6}$ & 63.78 & 55.07 & 60.00 \\
NaiveBayes & 77.22 & 56.25 & 54.05 & 59.84 & 56.04 & 52.60 \\
MLP & $\mathbf{8 0 . 3 8}$ & $\mathbf{6 4 . 5 8}$ & 60.36 & $\mathbf{6 5 . 7 5}$ & $\mathbf{6 7 . 6 3}$ & 64.11 \\
$k$-NN-2 & 70.25 & 54.17 & 54.05 & 57.48 & 60.39 & 60.00 \\
NNge & 76.58 & 62.50 & 62.16 & 60.24 & 59.42 & $\mathbf{6 6 . 8 5}$ \\
\hline
\end{tabular}

Note: SW, SeaWiFS OC; M-OC, MODIS OC; M-SST, MODIS SST; SWOC, SeaWiFS OC+ MODIS OC; MOD, MODIS OC+MODIS SST; All, OC+SST. Bold figures are maximum values. 
have enough samples, the algorithms produced accuracy rates equal or near 0 in those categories, lowering the mean considerably.

In view of the results, and since we did not have enough samples to improve them, it was decided to reduce the number of upwelling categories, grouping categories UA and UB in UAB, and categories UC and UD in UCD. This gave a total of 11 categories: six Upwelling (UAB, UBC, UCD, UABC, UBCD, and UABCD), two Upwelling Filament (UFAB and UFCD), Cool Eddy, Warm Eddy, and Wake. Table 3 lists the accuracy rates found using the different classification algorithms for the 11 MOS categories based on this new classification, and Table 4 details the descriptors selected for each test in this case.

Table 3. Results of MOS classification with the six tests for the 11 categories. All figures shown are percentages.

\begin{tabular}{lcccccc}
\hline Algorithm & SW & M-OC & M-SST & SWOC & MOD & All \\
\hline C 4.5 & 82.28 & 70.83 & 66.67 & 73.62 & 64.73 & 70.41 \\
NaiveBayes & 81.65 & 65.23 & 61.26 & 71.26 & 67.63 & 56.71 \\
MLP & 82.28 & 73.96 & $\mathbf{7 1 . 1 7}$ & 72.05 & $\mathbf{7 1 . 0 1}$ & 70.68 \\
$k$-NN-2 & 75.32 & 67.71 & 57.66 & 66.93 & 62.29 & 67.67 \\
NNge & $\mathbf{8 2 . 9 1}$ & $\mathbf{7 5 . 0 0}$ & 63.96 & $\mathbf{7 5 . 2 0}$ & 67.63 & $\mathbf{7 4 . 5 2}$ \\
\hline
\end{tabular}

Note: SW, SeaWiFS OC; M-OC, MODIS OC; M-SST, MODIS SST; SWOC, SeaWiFS OC+MODIS OC; MOD, MODIS OC+MODIS SST; All, OC+SST. Bold figures are maximum values.

Table 4. Selected descriptors. Tests used for the 11 categories of MOS.

\begin{tabular}{|c|c|c|c|c|c|c|}
\hline Descriptor & SW & $\mathrm{M}-\mathrm{OC}$ & M-SST & SWOC & MOD & All \\
\hline Perimeter & & & $\mathrm{X}$ & $\mathrm{X}$ & $\mathrm{X}$ & $X$ \\
\hline Area & $\mathrm{X}$ & $\mathrm{X}$ & $\mathrm{X}$ & $X$ & $\mathrm{X}$ & $X$ \\
\hline Centroid_ $x$ & & $X$ & $\mathrm{X}$ & & $X$ & $\mathrm{X}$ \\
\hline Centroid $y$ & $\mathrm{X}$ & & & $\mathrm{X}$ & & $\mathrm{X}$ \\
\hline MajorAxis & & & $\mathrm{X}$ & & $\mathrm{X}$ & $\mathrm{X}$ \\
\hline BBox_height & $\mathrm{X}$ & $\mathrm{X}$ & $\mathrm{X}$ & $\mathrm{X}$ & $\mathrm{X}$ & $\mathrm{X}$ \\
\hline BBox_width & & $\mathrm{X}$ & $\mathrm{X}$ & & $\mathrm{X}$ & $\mathrm{X}$ \\
\hline BBox_coord_ $x$ & & $\mathrm{X}$ & & & $X$ & \\
\hline BBox_coord $y$ & $X$ & & & $\mathrm{X}$ & & $\mathrm{X}$ \\
\hline $\mathrm{Hu} 2-$ & & & & $\mathrm{X}$ & & $\mathrm{X}$ \\
\hline Hu6 & & $\mathrm{X}$ & & & & \\
\hline MT11 & & $X$ & $\mathrm{X}$ & $\mathrm{X}$ & $\mathrm{X}$ & \\
\hline MT12 & & & & $\mathrm{X}$ & & \\
\hline MT21 & & $X$ & & & & \\
\hline MZ4 & & $\mathrm{X}$ & & & & \\
\hline Chl- $a$ _medium & $X$ & & & & & \\
\hline Cont_dis_near & $\mathrm{X}$ & $X$ & & $\mathrm{X}$ & $\mathrm{X}$ & $\mathrm{X}$ \\
\hline Cont_dis_medium & & $\mathrm{X}$ & & & & \\
\hline Island_dis_near & & & & & $X$ & \\
\hline Island_dis_medium & & $X$ & $\mathrm{X}$ & & $\mathrm{X}$ & \\
\hline Island_dis_far & & & $\mathrm{X}$ & $X$ & $X$ & $\mathrm{X}$ \\
\hline Size_medium & & $\mathrm{X}$ & & & & \\
\hline Size_big & & & & $\mathrm{X}$ & & \\
\hline
\end{tabular}

Note: SW, SeaWiFS OC; M-OC, MODIS OC; M-SST, MODIS SST; SWOC, SeaWiFS OC+MODIS OC; MOD, MODIS OC+MODIS SST; All, OC+SST. 
The results of each of the tests are described in Figures 6-11, which show the accuracy rate for each of the classifiers used based on the MOS subcategory to be classified. In these tests, it was shown that the use of the same descriptors decreases the classification accuracy rate, and thus different descriptors were used for each test (always over $60 \%$ relevance).

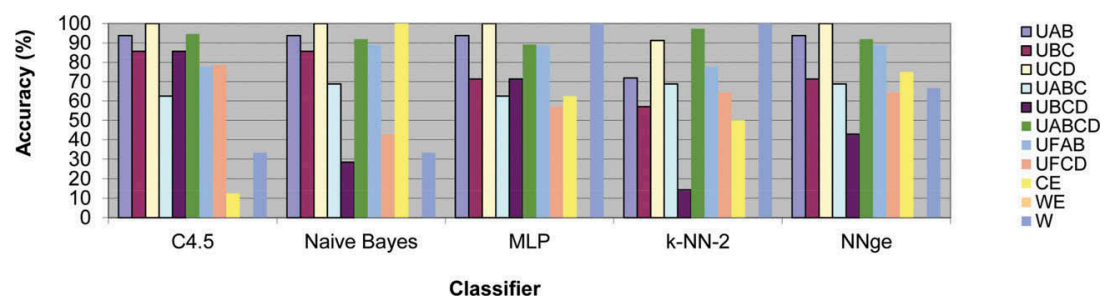

Figure 6. Accuracy rates for SeaWiFS OC sensor test, with 11 categories of MOS.

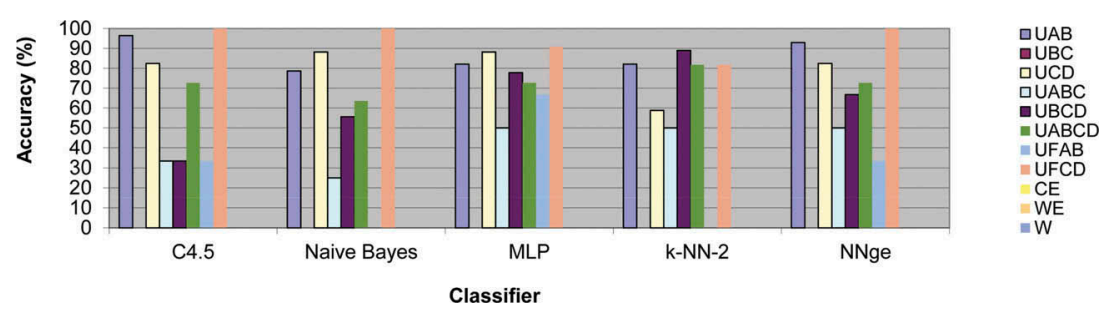

Figure 7. Accuracy rates for Aqua-MODIS OC sensor test, with 11 categories of MOS.

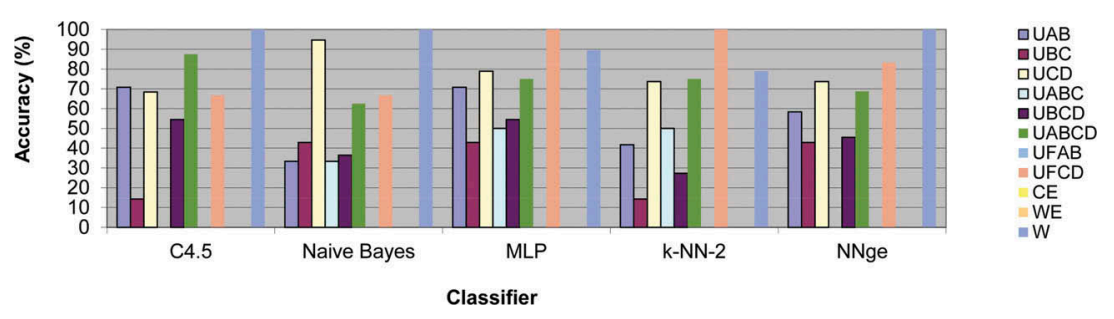

Figure 8. Accuracy rates for Aqua-MODIS SST sensor test, with 11 categories of MOS.

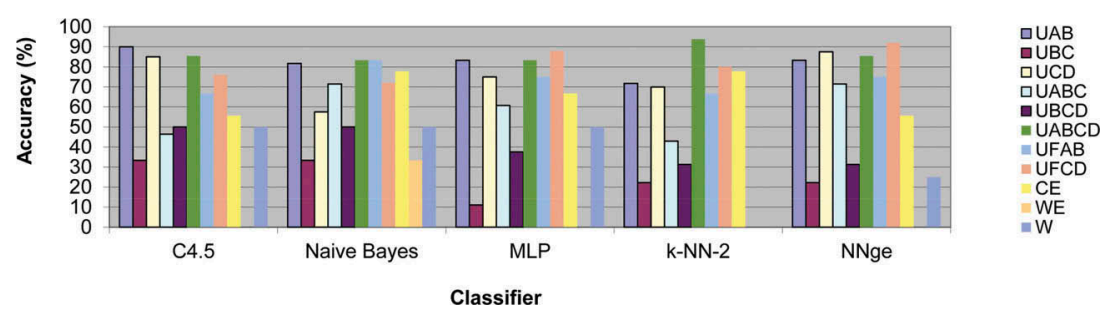

Figure 9. Accuracy rates for SeaWiFS OC and Aqua-MODIS OC sensors test, with 11 categories of MOS. 


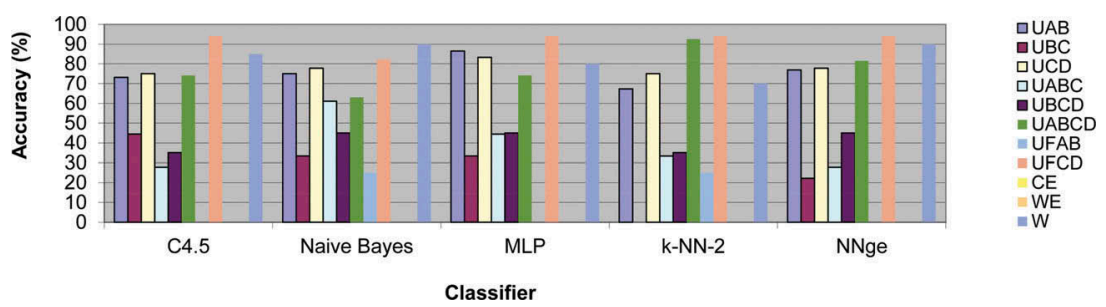

Figure 10. Accuracy rates for Aqua-MODIS OC and SST sensors test, with 11 categories of MOS.

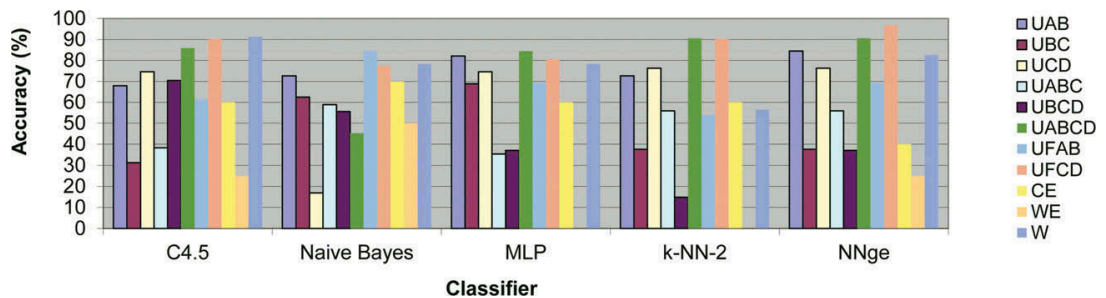

Figure 11. Accuracy rates for SeaWiFS OC, Aqua-MODIS OC, and SST sensors test, with 11 categories of MOS.

The sample of regions used for the SeaWiFS sensor (see Table 1) included 122 Upwellings (32 UAB, seven UBC, $23 \mathrm{UCD}, 16 \mathrm{UABC}$, seven UBCD, and 37 UABCD), 23 Upwelling Filaments (nine UFAB and 14 UFCD), eight Cool Eddies, two Warm Eddies, and three Wakes. Recall that for this classification in 11 categories, UA and UB were also counted in UAB, and UC and UD in UCD.

It is observed (see Figure 6) that the best classification was found with the NNge algorithm, with accuracy rates above $65 \%$ in most classes. The overall accuracy for this algorithm was $82.91 \%$, as shown in Table 3. In this case, the small sample of Warm Eddies (two) affected the results, since the classifiers had 0 hits for that class in all cases. On the other hand, there were a large number of UABCD, which increased the accuracy rate to $89 \%$ in all cases, as it did in the UCD.

The sample of regions used for the MODIS OC sensor (see Table 1) included 79 Upwellings (28 UAB, two UBC, $17 \mathrm{UCD}, 12 \mathrm{UABC}$, nine UBCD, and $11 \mathrm{UABCD}$ ), 14 Upwelling Filaments (three UFAB and 11 UFCD), one Cool Eddy, one Warm Eddy, and one Wake.

The best classification was found in this case (see Figure 7) with the NNge algorithm. The overall accuracy for this algorithm was $75 \%$, as shown in Table 3. Again, in this case the small sample of UBC (two), Cool Eddies (one), Warm Eddies (one), and Wakes (one) clearly affected the result, since the classifiers in these classes had 0 hits in all cases. Upwelling Filaments had the highest accuracy (100\% in most cases). The lack of samples makes the performance for Upwelling subcategories extremely irregular, except for UAB, which had an accuracy of over $78 \%$ in all cases.

The sample of regions used for the MODIS SST sensor (see Table 1) included 83 Upwellings (24 UAB, seven UBC, 19 UCD, six UABC, 11 UBCD, and 16 UABCD), seven Upwelling Filaments (one UFAB and six UFCD), one Cool Eddy, one Warm Eddy, and 19 Wakes. 
In this case, the best classification was found with the MLP algorithm (see Figure 8). The overall accuracy for this algorithm was $71.17 \%$, as shown in Table 3. As was the case with the previous test (MODIS OC), the small sample of UFAB (one), Cool Eddies (one), and Warm Eddies (one) clearly affected the result, since the classifiers had 0 hits for these classes in all cases. Accuracy for Wakes was $100 \%$ in most cases, since in this case there was a considerable sample. Something similar occurred with the UCD, with accuracy over $68 \%$ in all cases.

The sample of regions used for the test with regions from $\mathrm{OC}$ sensors (SeaWiFS and MODIS OC) included 201 upwellings (60 UAB, nine UBC, $40 \mathrm{UCD}, 28 \mathrm{UABC}, 16$ UBCD, and 48 UABCD), 37 Upwelling Filaments (12 UFAB and 25 UFCD), nine Cool Eddies, three Warm Eddies, and four Wakes.

The best classification was found with the NNge algorithm (see Figure 9). The overall accuracy for this algorithm was $75.20 \%$, as shown in Table 3. In this case, we had a larger sample of elements, especially in some categories, which raised the accuracy rate for them, as it did for UABCD, which had the highest accuracy (above $83 \%$ in all cases). However, the small sample of Warm Eddies (three) clearly affected the result, since the classifiers had 0 hits for that class in all cases save one (Naive-Bayes with 33.3\%).

The sample of regions used for the test with regions from the MODIS sensor, OC, and SST data included 162 Upwellings (52 UAB, nine UBC, 36 UCD, 18 UABC, 20 UBCD, and 27 UABCD), 21 Upwelling Filaments (four UFAB and 17 UFCD), two Cool Eddies, two Warm Eddies, and 20 Wakes.

In this case, the best classification was found with the MLP algorithm (see Figure 10). The overall accuracy for this algorithm was $71.01 \%$, as shown in Table 3 . Similar to the MODIS OC and MODIS SST tests separately, the small samples of Cool Eddies (two) and Warm Eddies (two) clearly affected the result, since the classifiers have 0 hits for those classes in all cases. This also happened with UFAB (four), which had 0 hits in most cases. Again, UFCD and Wakes show accuracy close to or over $90 \%$ in most cases.

The sample of regions used for the last test contained all the regions from the SeaWiFS and MODIS sensors, OC, and SST data, which included 284 Upwellings (84 $\mathrm{UAB}, 16 \mathrm{UBC}, 59 \mathrm{UCD}, 34 \mathrm{UABC}, 27 \mathrm{UBCD}$, and $64 \mathrm{UABCD}), 44$ Upwelling Filaments (13 UFAB and 31 UFCD), 10 Cool Eddies, four Warm Eddies, and 23 Wakes.

In the last test, it was observed (see Figure 11) how the best classification was found with the MLP algorithm. The overall accuracy for this algorithm was $74.52 \%$, as detailed in Table 3. As all of the samples were used, the results show accuracy rates above $70 \%$ in most cases, and for more categories: UAB, UCD, UABCD, UFCD, and Wakes.

Analysing the descriptors used in each of the tests as shown in Table 4, it may be seen how two, BBox_height and Area, are repeated in all of them. The descriptors Island_distance_near, Island_distance_medium, Island_distance_far, and Continent_distance near are also used in all of them, individually or together. These characteristics are decisive for distinguishing among some MOS categories and others, since the size and distance from the continent and islands are factors that distinguish, for instance, an upwelling from any other structure, and basically, also, for locating the upwelling (or piece of it) in Zones A, B, C, or D, or a filament farther to the north or south of our area of interest.

Analysing the data in Table 3, it may be seen how the overall accuracy in some cases is $82 \%$, and is over $65 \%$ in most cases. The algorithm that provided the best average results for all the tests was the MLP (mean 73.53\%). It may also be seen how the test conducted with the SeaWiFS sensor demonstrated the need for an MOS sample as varied and large as possible, since it provided better results than MODIS OC or MODIS SST. 
The morphological and contextual differences among the different categories make classification reasonably effective in this case with all the algorithms.

With the aim to qualitatively assess the performance of each classifier, Figure 12 visualizes an Aqua-MODIS SST scene with the MOS classified by an expert in different colours. The accuracy rate of C4.5, MLP, and NNge algorithms was $100 \%$, whereas the accuracy rate of Naïve Bayes was $80 \%$ because the UBCD (region number 2 in Figure 12) was erroneously classified as UABCD. Finally, the accuracy rate of the $k$ $\mathrm{NN}-2$ algorithm was $60 \%$ because the UBCD (region number 2 in Figure 12) was classified as UABCD and the wake-west of La Palma island (region number 3 in Figure 12) was classified as CE.

As an example of a characteristic error produced when one subcategory is classified as another, we have the possible classification of a cool eddy as an upwelling filament (UFAB or UFCD). Specifically, in the SeaWiFS sensor image in Figure 13, the similarity between a cool eddy (pink) and a possible upwelling filament in Zone CD can be noticed, since both MOS are characterized by having a high concentration of chl- $a$ (low SST) and being separated from the coast, and the cool eddy is a little

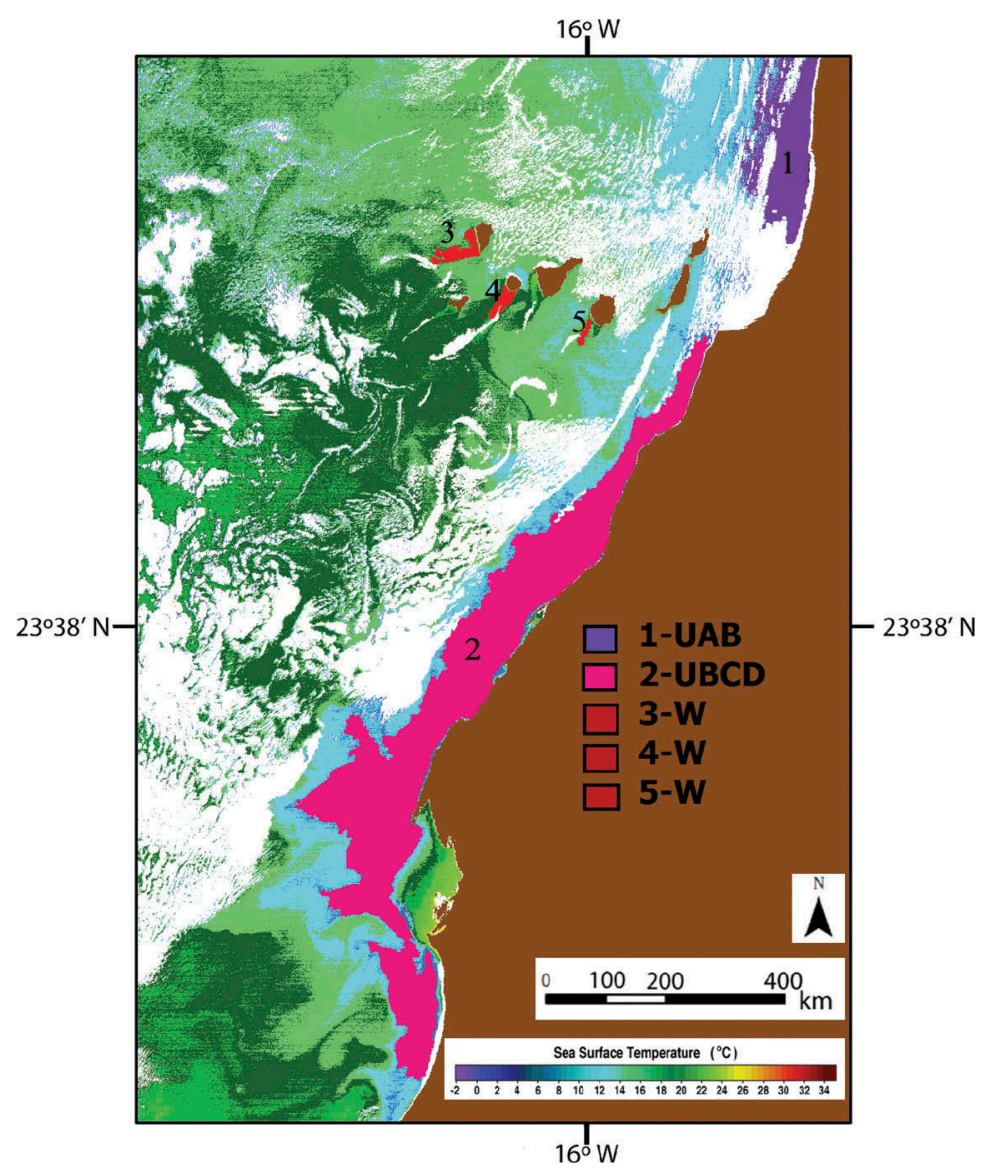

Figure 12. Aqua-MODIS SST scene (18 May 2009). MOS have been coloured and labelled for visualization: UAB (1) in lilac, UBCD (2) in pink, Wake-west of La Palma island (3) in red, WakeLa Gomera island (4) in red, and Wake-Gran Canaria island (5) in red. 


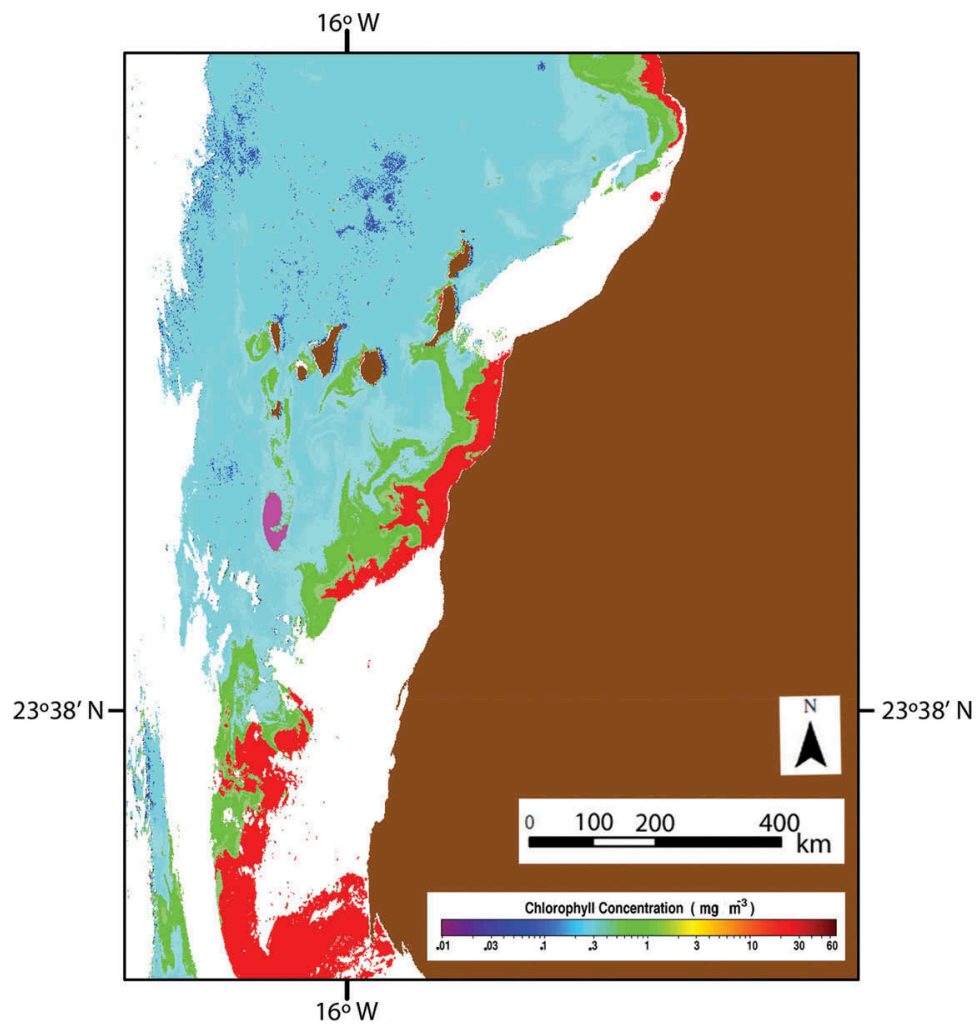

Figure 13. SeaWiFS sensor image (10 April 1999). Cool Eddy (pink colour).

longer than normal, which led to that classification error. In this case in particular, this error was caused with all five classification algorithms used.

\subsection{Comparison of results}

To test the robustness and improvement of the results over Vidal-Fernández et al. (2015), the confusion matrices of the new experiments (11 categories) were analysed to determine any deviations from the basic MOS categories (upwelling, upwelling filament, cool eddy, warm eddy, and wake), thereby reducing the number of categories to the five basic proposed in Vidal-Fernández et al. (2015). To perform this, we used the confusion matrices in the experiments conducted with 11 categories, with each of the classification algorithms used to determine which subcategories of upwellings and upwelling filaments were classified outside of the basic class (e.g. if an upwelling (UAB, UBCD, etc.) was classified as a subcategory other than an upwelling). The cool eddies, warm eddies, and wakes were classified directly as the basic class. Based on those data, comparative tables were made (Tables 5-10) in which the results are shown for each test with each classification algorithm. In each table, the results from our previous study are presented on the right and the new ones (extracted from the confusion matrices) are on the left. The results that improve or equal those of VidalFernández et al. (2015) are marked in bold. 
Table 5. Comparison of results with Vidal-Fernández et al. (2015). Accuracy rates of SeaWiFS sensor tests. All figures shown are percentages.

\begin{tabular}{lcccccccccc}
\hline MOS & C4.5 & Bayes & MLP & $k$-NN-2 & NNge & C4.5* & Naive Bayes* & MLP* & $k$-NN-2* & NNge* \\
\hline U & $\mathbf{1 0 0}$ & $\mathbf{1 0 0}$ & $\mathbf{1 0 0}$ & $\mathbf{1 0 0}$ & $\mathbf{1 0 0}$ & 100 & 96.70 & 100 & 100 & 100 \\
UF & 82.60 & $\mathbf{8 2 . 6 0}$ & 69.57 & 69.57 & 73.91 & 91.30 & 56.50 & 82.60 & 87.00 & 91.30 \\
CE & 12.50 & $\mathbf{1 0 0}$ & $\mathbf{6 2 . 5 0}$ & $\mathbf{5 0 . 0 0}$ & 75.00 & 87.50 & 100 & 50.00 & 50.00 & 87.50 \\
WE & $\mathbf{0}$ & $\mathbf{0}$ & 0 & 0 & 0 & 0 & 0 & 100 & 100 & 100 \\
W & 33.30 & $\mathbf{3 3 . 3 0}$ & $\mathbf{1 0 0}$ & $\mathbf{1 0 0}$ & $\mathbf{6 6 . 7 0}$ & 66.70 & 33.30 & 0 & 0 & 33.30 \\
\hline
\end{tabular}

Note: *Results of Vidal-Fernández et al. (2015); U, Upwelling; UF, Upwelling Filament; CE, Cool Eddy; WE, Warm Eddy; W, Wake. The results that improve or equal those of Vidal-Fernández et al. (2015) are marked in bold.

Table 6. Comparison of results with Vidal-Fernández et al. (2015). Accuracy rates of AquaMODIS OC sensor tests. All figures shown are percentages.

\begin{tabular}{lcccccccccc}
\hline & & Naive & & & & & & & & \\
MOS & C4.5 & Bayes & MLP & $k$-NN-2 & NNge & C4.5* & Naive Bayes* & MLP* & $k$-NN-2* & NNge* \\
\hline U & $\mathbf{1 0 0}$ & $\mathbf{1 0 0}$ & $\mathbf{1 0 0}$ & $\mathbf{1 0 0}$ & $\mathbf{1 0 0}$ & 100 & 98.70 & 100 & 100 & 100 \\
UF & 85.71 & $\mathbf{1 0 0}$ & 85.71 & 78.57 & $\mathbf{1 0 0}$ & 92.90 & 92.90 & 100 & 100 & 100 \\
CE & $\mathbf{0}$ & $\mathbf{0}$ & $\mathbf{0}$ & $\mathbf{0}$ & $\mathbf{0}$ & 0 & 0 & 0 & 0 & 0 \\
WE & $\mathbf{0}$ & $\mathbf{0}$ & $\mathbf{0}$ & $\mathbf{0}$ & $\mathbf{0}$ & 0 & 0 & 0 & 0 & 0 \\
W & $\mathbf{0}$ & $\mathbf{0}$ & $\mathbf{0}$ & $\mathbf{0}$ & $\mathbf{0}$ & 0 & 0 & 0 & 0 & 0
\end{tabular}

Note: *Results of Vidal-Fernández et al. (2015); U, Upwelling; UF, Upwelling Filament; CE, Cool Eddy; WE, Warm Eddy; W, Wake. The results that improve or equal those of Vidal-Fernández et al. (2015) are marked in bold.

Table 7. Comparison of results with Vidal-Fernández et al. (2015). Accuracy rates of AquaMODIS SST sensor tests. All figures shown are percentages.

\begin{tabular}{lcccccccccc}
\hline & & Naive & & & & & & & & \\
MOS & C4.5 & Bayes & MLP & $k$-NN-2 & NNge & C4.5* & Naive Bayes* & MLP* & $k$-NN-2* & NNge* \\
\hline U & $\mathbf{1 0 0}$ & 96.38 & 97.59 & 97.59 & 97.59 & 100 & 98.80 & 100 & 100 & 100 \\
UF & 42.86 & 42.86 & $\mathbf{8 5 . 7 1}$ & 85.71 & $\mathbf{7 1 . 4 3}$ & 57.10 & 85.70 & 71.40 & 100 & 57.10 \\
CE & $\mathbf{0}$ & $\mathbf{0}$ & $\mathbf{0}$ & $\mathbf{0}$ & $\mathbf{0}$ & 0 & 0 & 0 & 0 & 0 \\
WE & $\mathbf{0}$ & $\mathbf{0}$ & $\mathbf{0}$ & $\mathbf{0}$ & $\mathbf{0}$ & 0 & 0 & 0 & 0 & 0 \\
W & $\mathbf{1 0 0}$ & $\mathbf{1 0 0}$ & 89.50 & 78.90 & $\mathbf{1 0 0}$ & 94.70 & 94.70 & 94.70 & 89.50 & 89.50 \\
\hline
\end{tabular}

Note: *Results of Vidal-Fernández et al. (2015); U, Upwelling; UF, Upwelling Filament; CE, Cool Eddy; WE, Warm Eddy; W, Wake. The results that improve or equal those of Vidal-Fernández et al. (2015) are marked in bold.

For the SeaWiFS sensor test, the data in Table 5 show that the classification of upwellings, cool eddies, and wakes is improved (or equalled) with most of the classification algorithms. Classification was improved (or equalled) with the Naïve Bayes algorithm in all the categories.

For the Aqua-MODIS OC sensor test, the data in Table 6 show that upwelling classification was improved (or if not, equalled) by all the classification algorithms. 
Table 8. Comparison of results with Vidal-Fernández et al. (2015). Accuracy rates of SeaWiFS and Aqua-MODIS OC sensor tests. All figures shown are percentages.

\begin{tabular}{lcccccccccc}
\hline MOS & C4.5 & $\begin{array}{c}\text { Naive } \\
\text { Bayes }\end{array}$ & MLP & $k$-NN-2 & NNge & C4.5* & Naive Bayes* & MLP* & $k$-NN-2* & NNge* \\
\hline U & $\mathbf{1 0 0}$ & $\mathbf{9 9 . 0 0}$ & 99.00 & $\mathbf{1 0 0}$ & $\mathbf{1 0 0}$ & 100 & 97.50 & 100 & 100 & 100 \\
UF & 81.10 & $\mathbf{8 9 . 1 2}$ & 86.50 & 81.10 & $\mathbf{9 4 . 6 0}$ & 89.20 & 70.30 & 91.90 & 94.60 & 91.40 \\
CE & 55.60 & $\mathbf{7 7 . 8 0}$ & $\mathbf{6 6 . 7 0}$ & $\mathbf{7 7 . 8 0}$ & $\mathbf{5 5 . 6 0}$ & 66.70 & 55.60 & 33.30 & 33.30 & 44.40 \\
WE & 0 & $\mathbf{3 3 . 3 0}$ & 0 & 0 & 0 & 66.70 & 33.30 & 33.30 & 33.30 & 66.70 \\
W & 50.00 & $\mathbf{5 0 . 0 0}$ & 50.00 & 0 & 25.00 & 75.00 & 25.00 & 75.00 & 50.00 & 75.00 \\
\hline
\end{tabular}

Note: *Results of Vidal-Fernández et al. (2015); U, Upwelling; UF, Upwelling Filament; CE, Cool Eddy; WE, Warm Eddy; W, Wake. The results that improve or equal those of Vidal-Fernández et al. (2015) are marked in bold.

Table 9. Comparison of results with Vidal-Fernández et al. (2015). Accuracy rates of AquaMODIS OC and SST sensor tests. All figures shown are percentages.

\begin{tabular}{lcccccccccc}
\hline MOS & C4.5 & Naive & & & & & & & & \\
\hline U & $\mathbf{1 0 0}$ & 98.76 & 99.38 & 99.38 & $\mathbf{1 0 0}$ & 100 & 99.40 & 100 & 100 & 99.40 \\
UF & $\mathbf{8 1 . 0 0}$ & $\mathbf{8 5 . 7 1}$ & 80.95 & 80.95 & $\mathbf{8 5 . 7 1}$ & 81.00 & 85.70 & 95.20 & 90.50 & 85.70 \\
CE & $\mathbf{0}$ & $\mathbf{0}$ & $\mathbf{0}$ & $\mathbf{0}$ & $\mathbf{0}$ & 0 & 0 & 0 & 0 & 0 \\
WE & $\mathbf{0}$ & $\mathbf{0}$ & $\mathbf{0}$ & $\mathbf{0}$ & $\mathbf{0}$ & 0 & 0 & 0 & 0 & 0 \\
W & $\mathbf{8 5 . 0 0}$ & $\mathbf{9 0 . 0 0}$ & 80.00 & 70.00 & 90.00 & 75.00 & 90.00 & 95.00 & 80.00 & 95.00 \\
\hline
\end{tabular}

Note: *Results of Vidal-Fernández et al. (2015)); U, Upwelling; UF, Upwelling Filament; CE, Cool Eddy; WE, Warm Eddy; W, Wake. The results that improve or equal those of Vidal-Fernández et al. (2015) are marked in bold.

Table 10. Comparison of results with Vidal-Fernández et al. (2015). Accuracy rates of SeaWiFS and Aqua-MODIS OC and SST sensor tests. All figures shown are percentages.

\begin{tabular}{lcccccccccc}
\hline MOS & C4.5 & $\begin{array}{c}\text { Naive } \\
\text { Bayes }\end{array}$ & MLP & $k$-NN-2 & NNge & C4.5* & Naive Bayes* & MLP* & $k$-NN-2* & NNge* \\
\hline U & $\mathbf{9 9 . 6 5}$ & $\mathbf{9 9 . 2 9}$ & $\mathbf{9 8 . 9 4}$ & $\mathbf{1 0 0}$ & 99.65 & 99.30 & 78.20 & 98.90 & 99.60 & 100 \\
UF & $\mathbf{8 6 . 4 0}$ & $\mathbf{9 0 . 9 0}$ & 77.30 & 81.80 & $\mathbf{9 3 . 2 0}$ & 86.40 & 81.80 & 88.60 & 90.90 & 88.60 \\
CE & $\mathbf{6 0 . 0 0}$ & $\mathbf{7 0 . 0 0}$ & $\mathbf{6 0 . 0 0}$ & $\mathbf{6 0 . 0 0}$ & 40.00 & 60.00 & 70.00 & 50.00 & 40.00 & 60.00 \\
WE & $\mathbf{2 5 . 0 0}$ & $\mathbf{5 0 . 0 0}$ & 0 & 0 & 25.00 & 0 & 25.00 & 25.00 & 50.00 & 75.00 \\
W & $\mathbf{9 1 . 3 0}$ & 78.30 & 78.30 & 56.50 & 82.60 & 65.20 & 87.00 & 91.30 & 73.90 & 95.70 \\
\hline
\end{tabular}

Note: *Results of Vidal-Fernández et al. (2015); U, Upwelling; UF, Upwelling Filament; CE, Cool Eddy; WE, Warm Eddy; W, Wake. The results that improve or equal those of Vidal-Fernández et al. (2015) are marked in bold.

Improvements were made with the Naïve Bayes and NNge classification algorithms with $100 \%$ accuracy rates for upwellings and upwelling filaments. The rest of the categories were not classified due to lack of samples (one Cool Eddy, one Warm Eddy, and one Wake).

For the Aqua-MODIS SST sensor test, the data in Table 7 show that the classification of wakes improved with most of the classification algorithms. In addition, with the C4.5 and NNge classification algorithms, improvements were found in upwellings and 
upwelling filaments, respectively. The rest of the categories were not classified due to lack of samples (one Cool Eddy and one Warm Eddy).

For the SeaWiFS and Aqua-MODIS OC sensors test, the data in Table 8 show improvement in (or if not, equal to) the classification of upwellings and cool eddies by most of the classification algorithms. Classification was improved (or equalled) by the Naïve Bayes classification algorithm in all classes.

For the Aqua-MODIS OC and SST sensors test, the data in Table 9 show improvement in (or if not, equal to) classification of upwelling filaments with most of the classification algorithms. The $\mathrm{C} 4.5$ classification algorithm gave slightly better results, with a higher accuracy rate for wakes, and the rest remaining unchanged.

For the SeaWiFS and Aqua-MODIS OC and SST sensors test, the data in Table 10 show improvement in (or if not, equal to) the classification of upwellings, upwelling filaments, and cool eddies with most of the classification algorithms. With the C4.5 algorithm, improvements (or the same) were obtained in the classification of all categories. Substantial improvements were also obtained with the Naïve Bayes.

These results showed that not only were the deviations in classification not wider than in our previous study (Vidal-Fernández et al. (2015)), but for certain MOS categories, such as upwellings, cool eddies, and wakes, there were substantial improvements in classification. By choosing the best classification algorithm in each case, improvements can be obtained in all cases and in all categories.

Table 11 shows the percentage improvement for each test with each classification algorithm used, calculated by observing the number of categories in which the new approach has improved classification (e.g. if it improved in all five categories it would be $100 \%)$.

In the comparison performed, the classification algorithm that showed the best performance was the Naïve Bayes, which had an $86.6 \%$ mean improvement in the accuracy rate, followed by $\mathrm{C} 4.5$ with $73.3 \%$. Therefore, the new classification proposal (11 categories) not only matched the performance of the previous proposal in all five basic categories (Vidal-Fernández et al. (2015)) but also improved it and showed that the decision to create subcategories for upwellings (UA, UB, UC, UD, UAB, UBC, UCD, $\mathrm{UABC}, \mathrm{UBCD}$, and UABCD) and upwelling filaments (UFAB and UFCD) based on knowledge of the genesis and morphology of these MOS in the four zones of interest defined improved the accuracy rate, especially for upwellings, and enabled us to solve problems such as clouds and detailed monitoring, and their later analysis, thereby providing valuable information for oceanographers.

Table 11. Improvement in classification results over Vidal-Fernández et al. (2015).

\begin{tabular}{lccccc}
\hline & \multicolumn{5}{c}{ Algorithm } \\
\cline { 2 - 6 } Test & C4.5 & Naive Bayes & MLP & $k$-NN-2 & NNge \\
\hline SeaWiFS & $\mathbf{6 0}$ & $\mathbf{1 0 0}$ & $\mathbf{6 0}$ & $\mathbf{6 0}$ & 40 \\
MODIS OC & $\mathbf{8 0}$ & $\mathbf{1 0 0}$ & $\mathbf{8 0}$ & $\mathbf{8 0}$ & $\mathbf{1 0 0}$ \\
MODIS SST & $\mathbf{8 0}$ & $\mathbf{6 0}$ & $\mathbf{6 0}$ & 40 & $\mathbf{8 0}$ \\
SeaWiFS and MODIS OC & 20 & $\mathbf{1 0 0}$ & 20 & 40 & $\mathbf{6 0}$ \\
MODIS (OC and SST) & $\mathbf{1 0 0}$ & $\mathbf{8 0}$ & 40 & 40 & $\mathbf{8 0}$ \\
SeaWiFS and MODIS (OC and SST) & $\mathbf{1 0 0}$ & $\mathbf{8 0}$ & 40 & 40 & 20 \\
Mean improvement (\%) & $\mathbf{7 3 . 3}$ & $\mathbf{8 6 . 6}$ & $\mathbf{5 0 . 0}$ & $\mathbf{5 0 . 0}$ & $\mathbf{6 3 . 3}$ \\
\hline
\end{tabular}




\section{Conclusions}

This article presents a new approach to the classification of MOS present off the Northwest African coast based on their location, by dividing the area into four large zones based on their morphological characteristics, the seafloor topography and coastal relief. It has been shown that this division is not only consequent with the geographic variability, which clearly affects the genesis of MOS, but also in the ideal case of having exclusively upwelling regions belonging to only one of these zones, our system's performance is $100 \%$ accurate.

For the proposal of classification in 11 categories, the MLP algorithm provided the best average results for all the tests, with over $70 \%$ in all the experiments (mean $73.53 \%$ ). These results show that the work carried out to achieve effective segmentation providing significant final regions makes efficient automatic classification possible. When the sample of regions in all the categories is larger, results will improve considerably.

Results in the comparison with our previous study (Vidal-Fernández et al. (2015)) also showed that there were no wider deviations, and that substantial improvements in classification have been made for certain MOS categories, such as upwellings, cool eddies, and wakes. This ensures the robustness of our new proposal.

In practice, having to work with regions that span two or more zones with margins that are not defined a priori and not having a large enough sample worsened results. However, we have shown that our system can classify even under such unfavourable circumstances as these, so in future work, when improvements in segmenting tools and noise and cloud elimination allow, these accuracy rates will be around $100 \%$.

To be able to work with the SeaWiFS and Aqua-MODIS HDF files in other zones, such as certain areas of the North Atlantic and North Pacific that have wind and current conditions similar to those in our study area, and therefore have MOS similar to those classified, we have to modify our tool slightly, among other things, so that the filter in the starting position does not discard them. In principle, the tool should be able to give similar results, selecting the appropriate classification algorithms and with enough samples to ensure good training. This is precisely one of our next steps. Specifically, we want to be able to analyse NASA satellite L2 products with $1.1 \mathrm{~km}$ resolution acquired from the Oceancolor website, which uses the same flags.

All this work, in the long run, will enable the analysis of the location and evolution of the MOS over time and improve the prediction of its morphological evolution. In fact, in future work it is planned to make the tool able to automatically identify possible trends or changes in chl- $a$ concentration or the sea surface temperature analysing the morphology and evolution of MOS. At the same time we will be able to predict movement of the MOS by analysing their movement, routine, and the time they take to disappear.

\section{Disclosure statement}

No potential conflict of interest was reported by the authors.

\section{Funding}

This work was supported by the Spanish Ministry of Economy and Competitiveness (MINECO) [CGL2013-48202-C2-2-R,TIN2013-41576-R,TIN2013-44742-C4-4-R]; Andalusian Regional Government (Spain) [Project P10-TIC-6114]; CEiA3 and CEIMAR. 


\section{References}

Aha, D., D. Kibler, and M. K. Albert. 1991. "Instance-Based Learning Algorithms." Machine Learning 6: 37-66. doi:10.1007/BF00153759.

Angel, M. V., and M. J. R. Fasham. 1983. "Eddies and Biological Processes." In Eddies in Marine Science, edited by A. R. Robinson, 492-524. Berlin: Springer-Verlag.

Arístegui, J., P. Sangrá, S. Hernández-León, M. Cantón, A. Hernández-Guerra, and J. L. Kerling. 1994. "Island-Induced Eddies in the Canary Islands." Deep Sea Research Part I: Oceanographic Research Papers 41 (10): 1509-1525. doi:10.1016/0967-0637(94) 90058-2.

Baatz, M., C. Hoffmann, and G. Willhauck. 2008. "Progressing from Object-Based to ObjectOriented Image Analysis." In Object-Based Image Analysis, edited by T. Blaschke, S. Lang, and G. J. Hay, 29-42. Berlin: Springer.

Barton, E. D., J. Arístegui, P. Tett, M. Cantón, J. García-Braun, S. Hernández-León, L. Nykjaer, C. Almeida, J. Almunia, S. Ballesteros, G. Basterretxea, J. Escánez, L. García-Weill, A. Hernández-Guerra, F. López-Laatzen, R. Molina, M. F. Montero, E. Navarro-Pérez, J. M. Rodríguez, K. Van Lenning, H. Vélez, and K. Wild. 1998. "The Transition Zone of the Canary Current Upwelling Region." Progress In Oceanography 41 (4): 455-504. doi:10.1016/S0079-6611(98)00023-8.

Blaschke, T. 2010. "Object-Based Image Analysis for Remote Sensing." ISPRS Journal of Photogrammetry and Remote Sensing 65: 2-16. doi:10.1016/j.isprsjprs.2009.06.004.

Brent, M. 1995. Instance-Based learning: Nearest Neighbor With Generalization. New Zealand: Hamilton.

Brink, K. H. 1983. "The Near-Surface Dynamics of Coastal Upwelling.” Progress in Oceanography 12: 223-257. doi:10.1016/0079-6611(83)90009-5.

Climate Change 2013: The Physical Science Basis. 2013. Intergovernmental Panel On Climate Change AR4 WG1. USA: Cambridge Univ. Press.

Drăguț, L., and T. Blaschke. 2006. "Automated Classification of Landform Elements Using ObjectBased Image Analysis." Geomorphology 81 (3-4): 330-344. doi:10.1016/j. geomorph.2006.04.013.

Galvez, J. M., and M. Canton. 1993. "Normalization and Shape Recognition of Three-Dimensional Objects by 3D Moments.” Pattern Recognition 26 (5): 667-681. doi:10.1016/0031-3203(93) 90120-L.

García-Weil, L. 1998. "Descripción y análisis cuantitativo mediante series de imágenes de satélite de la dinámica de las aguas superficiales del noroeste de Africa." PhD diss., Univ. Las Palmas de Gran Canaria, Spain.

Garcia-Weil, L., M. Pacheco, G. Rodriguez, and T. McClimans. 2000. "Topographic Effects on the Mesoscale Ocean Circulation near Canarian Archipelago Examined by Means of Satellite Images and Laboratory Simulations." IEEE International Geoscience and Remote Sensing Symposium (IGARSS), Honolulu, HI, July 24-28, 1830-1832.

Gregg, W. W., M. E. Conkright, P. Ginoux, J. E. O'Reilly, and N. W. Casey. 2003. “Ocean Primary Production and Climate: Global Decadal Changes." Geophys Researcher Letters 30 (15): 18091812. doi:10.1029/2003GL016889.

Hall, M. A. 1998. Correlation-based Feature Subset Selection for Machine Learning. New Zealand: Hamilton.

Hernandez-Guerra, A., and L. Nykjaer. 1997. "Sea Surface Temperature Variability off North-West Africa: 1981-1989." International Journal of Remote Sensing 18: 2539-2558. doi:10.1080/ 014311697217468.

Hu, M. 1962. "Visual Pattern Recognition by Moment Invariants." IEEE Transactions on Information Theory 8: 179-187. doi:10.1109/TIT.1962.1057692.

John, G. H., and P. Langley. 1995. "Estimating Continuous Distributions in Bayesian Classifiers." In Eleventh Conference on Uncertainty in Artificial Intelligence, Montreal, 338-345. San Mateo: Morgan Kaufmann Publishers.

Jovanovic, D., M. Govedarica, I. Dordevic, and V. Pajic. 2010. "Object Based Image Analysis in Forestry Change Detection." IEEE 8th International Symposium on Intelligent Systems and Informatics, Subotica, September 10-11, 231-236.

Ko, B., and H. Byun. 2005. "FRIP: A Region-Based Image Retrieval Tool Using Automatic Image Segmentation and Stepwise Boolean and Matching." IEEE Transactions on Multimedia 7 (1): 105-113. doi:10.1109/TMM.2004.840603. 
Kohavi, R. 1995. "A Study of Cross-Validation and Bootstrap for Accuracy Estimation and Model Selection"." Proceedings of the Fourteenth International Joint Conference on Artificial Intelligence (San Mateo, CA: Morgan Kaufmann) 2 (12): 1137-1143.

Liu, Z., and Y. Hou. 2012. "Kuroshio Front in the East China Sea from Satellite SST and Remote Sensing Data." IEEE Geoscience and Remote Sensing Letters 9 (3): 517-520. doi:10.1109/ LGRS.2011.2173289.

Maitra, S. 1979. "Moment Invariants." In Proc. IEEE 67 (4): 697-699. doi:10.1109/ PROC.1979.11309.

Marcello, J., F. Marques, and F. Eugenio. 2005. "Automatic Tool for the Precise Detection of Upwelling and Filaments in Remote Sensing Imagery." IEEE Transactions on Geoscience and Remote Sensing 43 (7): 1605-1616. doi:10.1109/TGRS.2005.848409.

Meunier, T., D. Barton, B. Barreiro, and R. Torres. 2012. "Upwelling Filaments off Cap Blanc: Interaction of the NW African Upwelling Current and the Cape Verde Frontal Zone Eddy Field?" Journal of Geophysical Research: Oceans (1978-2012) 117: C8-031. doi:10.1029/ 2012JC007905.

Minsky, M., and S. Papert. 1972. Perceptrons: An Introduction to Computational Geometry. 2nd ed. Cambridge MA: The MIT Press.

Mityagina, M., and O. Lavrova. 2008. "Dynamic Phenomena in the Coastal Waters of the NorthEastern Black Sea Retrieved from Satellite Data." IEEE International Geoscience and Remote Sensing Symposium (IGARSS) 2: 347-350.

Musci, M., R. Queiroz Feitosa, and G. A. O. P. Costa. 2013. "An Object-Based Image Analysis Approach Based on Independent Segmentations." Proceedings of the Joint Urban Remote Sensing Event (JURSE), São Paulo, April 21-23, 275-278.

Nieto, K., H. Demarcq, and S. McClatchie. 2012. "Mesoscale Frontal Structures in the Canary Upwelling System: New Front and Filament Detection Algorithms Applied to Spatial and Temporal Patterns." Remote Sensing of Environment 123: 339-346. doi:10.1016/j. rse.2012.03.028.

Nykjaer, L. 1988. "Remote sensing applied to the Northwest African upwelling área." PhD diss., University of Copenhagen, Denmark.

Nykjaer, L., and L. Van-Camp. 1994. "Seasonal and Interannual Variability of Coastal Upwelling along Northwest Africa and Portugal from 1981 to 1991." Journal of Geophysical Research 99: 14197-14207. doi:10.1029/94JC00814.

Patel, S., R. Balasubramanian, and A. Gangopadhyay. 2008. "Automatic Detection of Oceanic Eddies in SeaWiFS-Derived Color Images Using Neural Networks and Shape Analysis." IEEE Geoscience and Remote Sensing Symposium (IGARSS) 2: 835-838.

Quinlan, R. 1993. C4.5: Programs for Machine Learning. San Mateo, CA: Morgan Kaufmann Publishers.

Rastner, P., T. Bolch, C. Notarnicola, and F. Paul. 2014. "A Comparison of Pixel-and Object-Based Glacier Classification With Optical Satellite Images." IEEE Journal of Selected Topics in Applied Earth Observations and Remote Sensing 7 (3): 853-862. doi:10.1109/ JSTARS.2013.2274668.

Rubino, A. 2005. Fluctuating Mesoscale Frontal Features: Structures and Manifestations in the Real Ocean. Hamburg, Germany: Kumulative Habilitationsschrift. Univ.

Sangra, P., J. L. Pelegrí, A. Hernández-Guerra, I. Arregui, J. M. Martín, A. Marrero-Díaz, A. Martínez, A. W. Ratsimandresy, and A. Rodríguez-Santana. 2005. "Life History of an Anticyclonic Eddy." Journal of Geophysical Research 110 (19): C3-021. doi:10.1029/ 2004JC002526.

Sathyendranath, S., B. Brewin, D. Mueller, R. Doerffer, H. Krasemann, F. Mélin, C. Brockmann, P. et al. 2012. "Ocean Colour Climate Change Initiative-Approach and Initial Results." IEEE International Geoscience and Remote Sensing Symposium (IGARSS), Munich, July 22-27, 2024-2027.

Saulquin, B., F. Gohin, and R. Garrello. 2011. "Regional Objective Analysis for Merging HighResolution MERIS, Modis/Aqua, and SeaWiFS Chlorophyll-A Data from 1998 to 2008 on the European Atlantic Shelf." IEEE Transactions on Geoscience and Remote Sensing 49 (1): 143154. doi:10.1109/TGRS.2010.2052813.

Shrivastava, N., and V. Tyagi. 2014. "A Review of ROI Image Retrieval Techniques." Proceedings of the 3rd International Conference on Frontiers of Intelligent Computing: Theory and Applications (FICTA), Springer 2: 509-520. 
Teague, M. R. 1980. "Image Analysis via the General Theory of Moments." Journal of the Optical Society of America 70 (8): 920-930. doi:10.1364/JOSA.70.000920.

Tejera, A., L. García-Weil, K. Heywood, and M. Cantón-Garbín. 2002. "Observations of Oceanic Mesoscale Features and Variability in the Canary Islands Area from ERS-1 Altimeter Data, Satellite Infrared Imagery and Hydrographic Measurements." International Journal of Remote Sensing 23 (22): 4897-4916. doi:10.1080/01431160110034681.

Troupin, C., E. Mason, J.-M. Beckers, and P. Sangrà. 2012. "Generation of the Cape Ghir Upwelling Filament: A Numerical Study." Ocean Modelling 41: 1-15. doi:10.1016/j. ocemod.2011.09.001.

Van-Camp, L., L. Nykjaer, E. Mittelstaedt, and P. Schlittenhardt. 1991. "Upwelling and Boundary Circulation off Northwest Africa as Depicted by Infrared and Visible Satellite Observations." Progress in Oceanography 26: 357-402. doi:10.1016/0079-6611(91)90012-B.

Vidal-Fernández, E., J. A. Piedra-Fernández, J. M. Almendros-Jiménez, and M. Cantón-Garbín. 2015. "OBIA System for Identifying Mesoscale Oceanic Structures in SeaWiFS and MODISAqua Images." IEEE Journal of Selected Topics in Applied Earth Observations and Remote Sensing 8 (3): 1256-1265.

Xiao, B., S. Hu, and X. Qiang. 2010. "Research on the Ocean Primary Production Pattern Based Remote Sensing." IEEE International Conference on Audio Language and Image Processing (ICALIP), Shanghai, November 23-25, 1543-1546.

Zunic, J., and N. Sladoje. 2000. "Efficiency of Characterizing Ellipses and Ellipsoids by Discrete Moments." IEEE Transactions on Pattern Analysis and Machine Intelligence 22 (4): 407-414. doi: $10.1109 / 34.845384$. 